\title{
Distractive Auditory Stimuli Alleviate the Perception of Dyspnea Induced by Low-Intensity Exercise in Elderly Subjects With COPD
}

\author{
Kazuya Shingai PT MSc, Masashi Kanezaki PT PhD, and Hideaki Senjyu PT PhD
}

\begin{abstract}
BACKGROUND: Although recent studies have shown that distractive auditory stimuli (DAS) in the form of music increase adherence to exercise in subjects with COPD, the effect of DAS on dyspnea induced by low-intensity, constant-load exercise in elderly patients with COPD has not been elucidated. Therefore, the purpose of this study was to investigate the effect of DAS on the perception of dyspnea induced by low-intensity, constant-load exercise in elderly subjects with COPD. METHODS: We enrolled 16 male out-patients with COPD. Subjects completed cycling exercises with and without DAS at $40 \%$ maximum oxygen consumption. They were asked to rate their perception of dyspnea using the modified Borg scale every 3 min during exercise and every 1 min during the recovery period. RESULTS: Dyspnea perception during low-intensity exercise showed a significant correlation between the exercise condition (DAS and control) and exercise duration $(P=.04)$. Exercise-induced dyspnea perception under the DAS condition was significantly lower than that under the control condition from $18 \mathrm{~min}$ after the start of exercise to $3 \mathrm{~min}$ after the end of exercise $(18,20,21,22$, and $23 \mathrm{~min}, P=.01, P<.001, P=.009, P=.006$, and $P=.006$, respectively). However, oxygen consumption and ventilation in response to low-intensity exercise did not significantly differ in the DAS and control conditions $(P=.39$ and .14 , respectively $)$ CONCLUSIONS: Our results suggest that DAS is a non-pharmacologic therapy that can be used to reduce the dyspneic sensation in elderly patients with COPD. Key words: distractive auditory stimuli; exercise-induced dyspnea; COPD; elderly; low-intensity exercise; cycle ergometer. [Respir Care 2015;60(5):689-694. (C) 2015 Daedalus Enterprises]
\end{abstract}

\section{Introduction}

Dyspnea, which is often defined as an uncomfortable awareness of difficulty in breathing, is not only a subjective sensation but also a serious respiratory symptom in patients with COPD. In particular, during COPD exacerbations, patients experience an excessive perception of

\footnotetext{
Mr Shingai is affiliated with the Department of Rehabilitation Medicine, Tosei General Hospital, Seto, Aichi, Japan. Dr Kanezaki is affiliated with the Department of Physical Therapy, Faculty of Health Care Sciences, Himeji Dokkyo University, Himeji, Hyogo, Japan. Dr Senjyu is affiliated with the Department of Cardiopulmonary Rehabilitation Science, Nagasaki University Graduate School of Biomedical Sciences, Nagasaki, Japan.

This study was supported by Grants-in-Aid for Scientific Research 24500589 and 26870760 from the Ministry of Education, Culture, Sports, Science, and Technology and by Kirigaoka-Tsuda Hospital, the Nagasaki Pulmonary Rehabilitation Clinic, and Tagami Hospital. The authors have disclosed no conflicts of interest.
}

dyspnea beyond normal day-to-day variations. This excessive perception of dyspnea during COPD exacerbations generally leads to a limitation of weight-bearing daily activities requiring the recruitment of many motor units. ${ }^{1} \mathrm{We}$ previously found that the perception of dyspnea induced by breath-holding has an inhibitory effect on the muscular strength of stable subjects with COPD. ${ }^{1}$ Therefore, reduction of unpleasant respiratory sensations can play a crucial role in preventing physical inactivity in patients with COPD.

It has been reported that distractive auditory stimuli (DAS) in the form of music can decrease the perception of

\footnotetext{
Correspondence: Masashi Kanezaki PT PhD, Department of Physical Therapy, Faculty of Health Care Sciences, Himeji Dokkyo University, 7-2-1 Kamiohno, Himeji, Hyogo 670-8524, Japan. E-mail: kmasashi@ gm.himeji-du.ac.jp.
}

DOI: $10.4187 /$ respcare.03533 
dyspnea induced by exercise in subjects with COPD. ${ }^{2}$ In addition, DAS has increased adherence to exercise in subjects with COPD. ${ }^{3}$ Thus, to maintain a suitable degree of physical activity, DAS during physical exercise seem to be a useful tool in stable patients with COPD. However, the mechanism of the inhibitory effect of DAS on dyspnea induced by exercise has not been fully elucidated.

A cycle ergometer is one of the most common tools used in exercise testing and intervention in patients with COPD ${ }^{4}$ Previous studies that investigated the inhibitory effect of DAS on dyspnea induced by exercise used treadmills and field-walking tests. ${ }^{2,3}$ However, it is recognized that cycle ergometer exercise can more accurately maintain the intensity of exercise load compared with either treadmill exercise or field walking. ${ }^{4,5}$ In addition, it has been reported that there is a difference in the perception of dyspnea experienced by subjects using treadmills versus cycle ergometers. ${ }^{6}$ However, the effect of DAS on the perception of dyspnea induced by cycle ergometer exercise in patients with COPD has not been clarified.

The perception of dyspnea induced by low-intensity exercise occurs often during many activities of daily living in patients with COPD. ${ }^{7}$ Yamashita et $\mathrm{al}^{8}$ investigated the effect of listening to music on dyspnea perception during exercise at $40 \%$ and $60 \%$ maximum oxygen consumption $\left(\dot{\mathrm{V}}_{\mathrm{O}_{2}}\right)$ in healthy adult males. They found that music decreased the dyspneic sensation during exercise at $40 \%$ maximum $\dot{\mathrm{V}}_{\mathrm{O}_{2}}$, but not at $60 \%$ maximum $\dot{\mathrm{V}}_{\mathrm{O}_{2}}$. Thus, although use of DAS to counter dyspnea induced by lowintensity exercise in patients with COPD can play a crucial role in preventing physical inactivity, its effect on dyspnea induced by low-intensity, constant-load exercise in patients with COPD remains unclear.

Dyspnea is the result of sensory activation of subcortical and cortical neural pathways. Some of these pathways are shared across respiratory modalities, whereas activation of some neural areas is modality-specific. ${ }^{9}$ Many brain imaging studies of dyspnea have been conducted using different techniques to induce dyspnea. Despite the use of different intervention techniques, a common predominant neural activity has been found in the insula, operculum, and frontal cortex areas; anterior and posterior cingulated cortices; cerebellum; thalamus; and amygdala. ${ }^{9,10}$ However, a common predominant activity during auditory stimuli has been found in the primary and secondary auditory cortices and insular cortex. ${ }^{11}$ Because the cortical processing involved in auditory stimuli is partly consistent with that in dyspnea perception, ${ }^{9-11}$ the respiratory peripheral neural afferents of dyspnea perception, activated by exercise, may interact with the neural circuit responsible for processing dyspnea.

It is recognized that when 2 nerve impulses occur on a common neural substrate, the net resulting intensity of the response will be lower than that expected from the sum-

\section{QUICK LOOK}

\section{Current knowledge}

Dyspnea is a subjective sensation and a serious respiratory symptom in patients with COPD. This excessive perception of dyspnea during COPD exacerbation leads to limitation of weight-bearing daily activities. Distractive auditory stimuli (DAS) in the form of music have been shown to decrease the perception of dyspnea induced by exercise in patients with COPD.

\section{What this paper contributes to our knowledge}

The results indicate that DAS reduces the perception of dyspnea induced by low-intensity, constant-load exercise in elderly subjects with COPD. DAS is a nonpharmacologic therapy that can be used to reduce the dyspneic sensation in COPD following exercise. The impact during a COPD exacerbation remains to be determined.

mation of the responses of the 2 separate stimuli. This physiological phenomenon has been termed neural occlusion. ${ }^{12}$ Because the central processing area for dyspnea perception and auditory stimuli is partly shared, ${ }^{9-11}$ the perception of dyspnea induced by exercise could be interfered with by auditory stimuli in elderly patients with COPD. Thus, low-intensity exercise-induced dyspnea perception can be modified by DAS in patients with COPD.

In addition, because previous studies showed that with an increase in age, subjects become less sensitive to dyspnea and auditory stimuli, ${ }^{13,14}$ the inhibitory effect of DAS on dyspnea could be modified by age. However, there are no data on the effect of DAS on dyspnea in elderly patients with COPD. Therefore, our aim was to investigate the effect of DAS on dyspnea induced by low-intensity, constant-load exercise in elderly subjects with COPD.

\section{Methods}

\section{Subjects}

The subjects were 16 male out-patients with COPD who were referred to the pulmonary rehabilitation programs at Kirigaoka-Tsuda Hospital, the Nagasaki Pulmonary Rehabilitation Clinic, and Tagami Hospital in Nagasaki, Japan. They were eligible to participate if they were under the care of a respiratory physician and were ambulant and clinically stable, with no changes in medication for at least 4 weeks before recruitment.

A diagnosis of COPD was made according to published guidelines. ${ }^{15}$ Exclusion criteria were orthopedic or neuro- 


\section{EfFect of DAS on Dyspnea in Elderly Subjects With COPD}

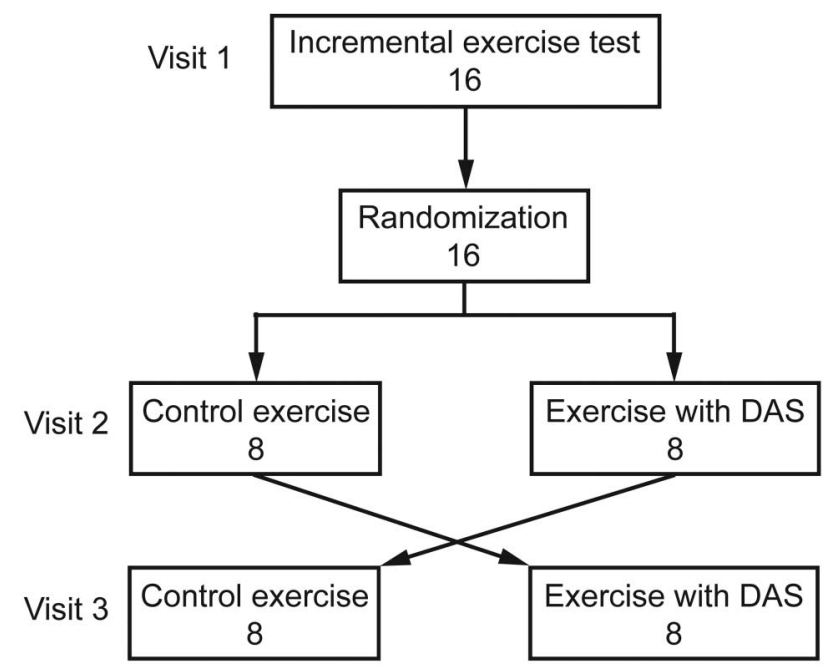

Fig. 1. Flow chart. DAS = distractive auditory stimuli.

logical disorders limiting exercise performance, unstable cardiac disease, supplemental oxygen therapy, arterial hypercapnia $\left(\mathrm{P}_{\mathrm{aCO}_{2}}>45 \mathrm{~mm} \mathrm{Hg}\right)$, and severe hearing impairment. All subjects provided informed written consent before data collection, and the study was approved by the institutional review board of the Nagasaki University Graduate School of Biomedical Sciences.

\section{Experimental Protocol}

The subjects were in the laboratory for $3 \mathrm{~d}$ within a 2-week period; experimental sessions were separated by a 24-h interval. All subjects were entered into a randomized crossover study to compare non-DAS and DAS conditions (Fig. 1). The subjects were given a short training period to accustom them to the apparatus and the use of the modified Borg scale.

\section{DAS}

The type of music selected by individual subjects included folk songs, old songs, and popular music. The volume of music was individually adjusted by the subjects before the test. The tempo of music selected was moderate to fast. Under the DAS condition, the subjects exercised on a cycle ergometer while listening to music via earphones on a portable MP3 player. Under the control condition, subjects wore earphones, but no music was played.

\section{Exercise Testing}

All sessions took place in a quiet laboratory. On the first day of testing, subjects performed a standardized incremental exercise test using an electromagnetically braked cycle ergometer (232CXL, Combi, Tokyo, Japan) accord- ing to the American Thoracic Society/American College of Chest Physicians guidelines. ${ }^{4}$ During exercise testing, $\dot{\mathrm{V}}_{\mathrm{O}_{2}}$, carbon dioxide production, minute ventilation $\left(\dot{\mathrm{V}}_{\mathrm{E}}\right)$, and breathing frequency were measured by the breath-bybreath method using AT1000 3.0 (Anima, Tokyo, Japan). The mean value of oxygen gas and $\dot{V}_{\mathrm{E}}$ during 1 min was used for analysis. The incremental exercise test consisted of $3 \mathrm{~min}$ of rest and $3 \mathrm{~min}$ of unloaded pedaling at $60 \mathrm{rpm}$, followed by a progressive increase in work load of 10 $\mathrm{W} / \mathrm{min}$ until exhaustion.

On the second and third days, subjects performed experimental constant-load cycling at $40 \%$ maximum $\dot{\mathrm{V}}_{\mathrm{O}_{2}}$ with and without DAS, respectively. The experimental protocol consisted of a 5-min seated rest period, followed by a 20-min cycling exercise and a 5-min recovery period. The subject rated the sensation of dyspnea using the modified Borg scale every $3 \mathrm{~min}$ during exercise and every 1 min during the recovery period, selecting a number from a scale of 0 (no dyspnea) to 10 (maximum dyspnea). At the beginning of the measurement, we asked each subject to rate the sensation of kokyu-konnan (discomfort of breathing). This term is an exact Japanese translation of dyspnea: kokyu means breathing or respiration, and konnan means discomfort or difficulty. Kokyu-konnan was not defined any further, but the subjects were instructed to avoid rating non-respiratory sensations, such as headache or pharynx irritation. ${ }^{16}$

\section{Statistical Analysis}

Data are presented as mean $\pm \mathrm{SD}$. Under both the DAS and control conditions, the modified Borg scale and expired gas analysis data during constant-load cycling were analyzed using 2-way analysis of variance with repeated measures. The 2 factors were experimental conditions (DAS and control) and exercise duration $(0,3,6,9,12,15,18$, $20,21,22,23,24$, and $25 \mathrm{~min}$ ). In addition, the differences in the modified Borg scale at equivalent exercise times (ie, isotime for each subject) between the DAS and control conditions were analyzed using a paired $t$ test. All analyses were calculated with SPSS 15.0 (SPSS, Chicago, Illinois). $P<.05$ was considered significant.

\section{Results}

All 16 subjects completed the experiments without any difficulty or side effects. Subject characteristics are summarized in Table 1. Table 2 shows the exercise capacity characteristics. Figure 2 shows the time courses of exercise-induced dyspnea under both the DAS and control conditions. Dyspnea perception during low-intensity exercise showed a significant correlation between exercise condition (DAS and control) and exercise duration $(P=.04)$. As shown in Figure 2, exercise-induced dyspnea percep- 
Table 1. Characteristics of Subjects With COPD

\begin{tabular}{|c|c|}
\hline Characteristics & Values \\
\hline Age, y & $75.7 \pm 8.4$ \\
\hline Weight, kg & $55.1 \pm 8.0$ \\
\hline Height, $\mathrm{cm}$ & $159.9 \pm 8.4$ \\
\hline BMI, $\mathrm{kg} / \mathrm{m}^{2}$ & $21.5 \pm 2.8$ \\
\hline FVC, L & $2.50 \pm 0.87$ \\
\hline FVC, \% predicted & $78.1 \pm 25.5$ \\
\hline $\mathrm{FEV}_{1}, \mathrm{~L}$ & $1.41 \pm 0.6$ \\
\hline $\mathrm{FEV}_{1}, \%$ predicted & $53.6 \pm 19.8$ \\
\hline $\mathrm{FEV}_{1} / \mathrm{FVC}, \%$ & $52.3 \pm 16.3$ \\
\hline MRC scale, $n(0 / 1 / 2 / 3 / 4)$ & $0 / 9 / 4 / 3 / 0$ \\
\hline $\begin{array}{l}\text { COPD severity according to GOLD standards, } \\
n(1 / 2 / 3 / 4 / 5)\end{array}$ & $2 / 6 / 7 / 4 / 0$ \\
\hline $\begin{array}{l}\text { Data are mean } \pm \text { SD. } \\
\text { BMI }=\text { body mass index } \\
\text { MRC }=\text { Medical Research Council } \\
\text { GOLD }=\text { Global Initiative for Chronic Obstructive Lung Disease }\end{array}$ & \\
\hline
\end{tabular}

Table 2. Characteristics of Exercise Capacity

\begin{tabular}{lc}
\hline \hline \multicolumn{1}{c}{ Characteristics } & Values \\
\hline 6MWT & \\
$\quad$ Distance, $\mathrm{m}$ & $389.4 \pm 79.1$ \\
$\quad$ Modified Borg dyspnea scale after exercise & $4.3 \pm 2.3$ \\
Cardiopulmonary exercise test & \\
$\quad$ Maximum $\dot{\mathrm{V}}_{\mathrm{O}_{2}}, \mathrm{~mL} / \mathrm{kg} / \mathrm{min}$ & $16.8 \pm 3.7$ \\
Peak heart rate, beats/min & $116.3 \pm 17.5$ \\
Peak load, $\mathrm{W}$ & $68.6 \pm 21.2$ \\
$\dot{\mathrm{V}}_{\mathrm{E}} / \mathrm{MVV}, \%$ & $80.2 \pm 28.4$ \\
Modified Borg dyspnea scale after exercise & $6.1 \pm 1.6$ \\
& \\
\hline Data are mean \pm SD. & \\
$6 \mathrm{MWT}=6$-min walk test & \\
$\dot{\mathrm{V}}_{\mathrm{O}_{2}}=$ oxygen consumption & \\
$\dot{\mathrm{V}}_{\mathrm{E}}=$ minute ventilation \\
MVV = maximum voluntary ventilation \\
\hline
\end{tabular}

tion under the DAS condition was significantly lower than that under the control condition from 18 min after the start of exercise to $3 \mathrm{~min}$ after the end of exercise $(P=.01$, $P<.001, P=.009, P=.006$, and $P=.006$, respectively). However, as shown in Figures 3 and $4, \dot{\mathrm{V}}_{\mathrm{O}_{2}}$ and $\dot{\mathrm{V}}_{\mathrm{E}}$ in response to low-intensity exercise did not significantly differ between the DAS and control conditions $(P=.39$ and .14 , respectively).

\section{Discussion}

In this study, we found that DAS lessened the perception of dyspnea induced by low-intensity, constant-load exercise on a cycle ergometer in elderly subjects with COPD. In addition to this phenomenon, the effect of DAS on dyspnea increased with increasing exercise duration.

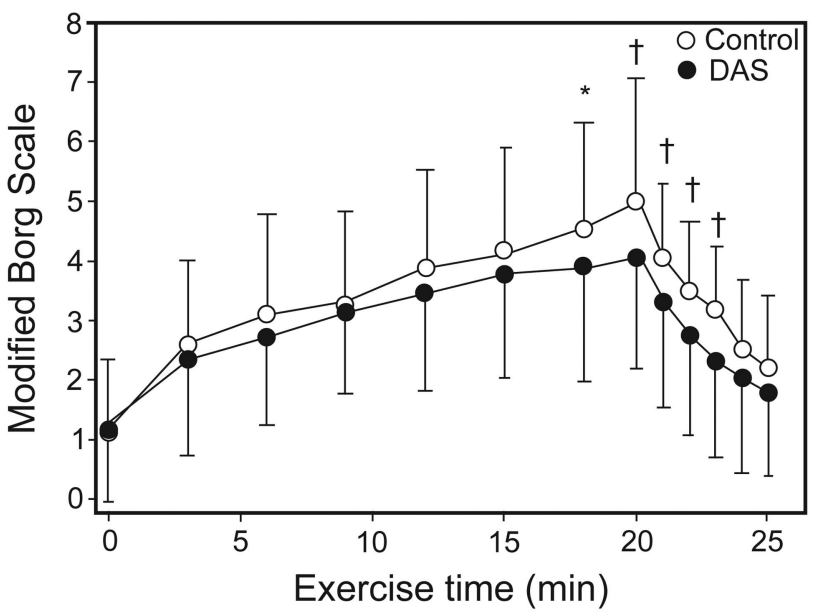

Fig. 2. Time courses of exercise-induced dyspnea under both the distractive auditory stimuli (DAS) and control conditions. Dyspnea was measured using the modified Borg scale. Data are shown as mean $\pm \mathrm{SD} .{ }^{*} P<.05 ; \dagger P<.01$.

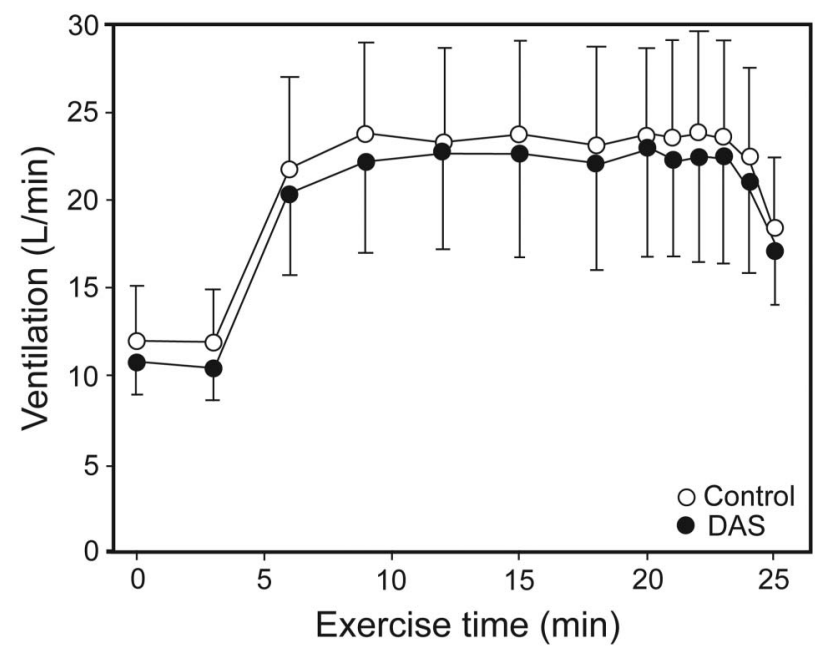

Fig. 3. Time courses of minute ventilation in response to lowintensity exercise under both the distractive auditory stimuli (DAS) and control conditions. Data are shown as mean \pm SD.

However, $\dot{\mathrm{V}}_{\mathrm{O}_{2}}$ and $\dot{\mathrm{V}}_{\mathrm{E}}$ did not significantly differ between the DAS and control conditions.

There have been several studies on the effect of music on exercise-induced dyspnea. ${ }^{2,3,17,18}$ Our observations in this study are consistent with those in previous studies that investigated the effect of DAS on dyspneic sensation induced by exercise. ${ }^{2,17}$ Thornby et al ${ }^{17}$ showed that DAS attenuated treadmill exercise-induced dyspnea. von Leupoldt et $\mathrm{al}^{2}$ reported that DAS decreased dyspnea during a 6-min walk test in subjects with COPD. These studies clearly show the role of DAS in the perception of dyspnea induced by walking. ${ }^{2,17}$ However, due to a lack of data on consecutive measurements of perception of dyspnea induced by exercise, ${ }^{2,17}$ the effect of DAS on the time-dependent 


\section{EfFect of DAS on Dyspnea in Elderly Subjects With COPD}

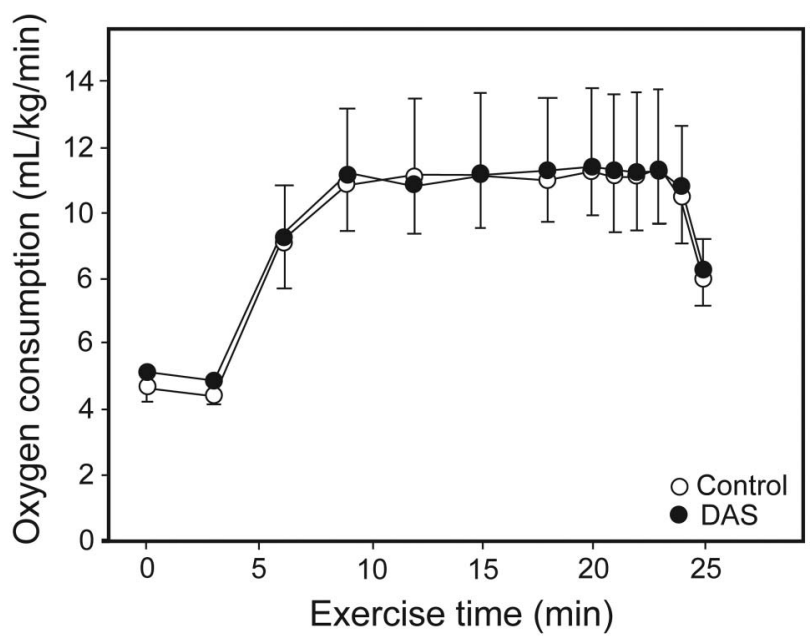

Fig. 4. Time courses of oxygen consumption in response to lowintensity exercise under both the distractive auditory stimuli (DAS) and control conditions. Data are shown as mean $\pm \mathrm{SD}$.

changes in dyspnea in response to exercise load in subjects with COPD has not been elucidated. In this study, we showed that the effect of DAS on dyspnea increased with increasing exercise duration. This phenomenon has not been reported in other studies on the effect of DAS on dyspnea in subjects with COPD. . $^{2,317,18}$ This novel finding suggests that DAS may enable patients with COPD to exercise for longer time periods.

Cycle ergometer exercise can more accurately maintain the intensity of exercise load than either treadmill exercise or field walking. ${ }^{4}$ In this study, the intensity of exercise used was lower than that in previous studies investigating the effect of DAS on exercise-induced dyspnea. ${ }^{2,17} \mathrm{Be}$ cause the perception of dyspnea induced by low-intensity exercise often occurs in many activities of daily living in patients with COPD, ${ }^{7}$ the results of our study may contribute to the usefulness of DAS to lessen dyspnea perception during low-intensity, daily physical exercise in patients with COPD.

The subjects in this study were elderly patients with COPD, in contrast to previous studies that used younger subjects to investigate the effect of DAS on exercise-induced dyspnea. ${ }^{2,17}$ Because previous studies showed that subjects become less sensitive to dyspnea and auditory stimuli with increased age, ${ }^{13,14}$ the inhibitory effect of DAS on dyspnea can be modified by age. However, in this study, we demonstrated that the perception of dyspnea induced by constant-load exercise was clearly reduced by DAS in elderly subjects with COPD, which agreed with findings of previous studies.,2,17 Therefore, our data suggest that the perception of dyspnea induced by exercise can be reduced by DAS in elderly patients with COPD.

When 2 or more nerve impulses occur on a common neural substrate, the net resulting intensity of response will be lower than that expected from summation of the responses to the 2 stimuli acting separately. This physiological phenomenon has been termed neural occlusion. ${ }^{12} \mathrm{Be}-$ cause the central processing area for dyspnea perception and auditory stimuli is partly shared, the perception of dyspnea induced by exercise can be interfered with by auditory stimuli in elderly patients with COPD.

In this study, dyspnea was induced by 20 min of lowintensity, constant-load exercise. The most serious limitation of this study is the lack of data to compare lowintensity and high-intensity exercise. This prevented us from investigating whether the effect of DAS on dyspnea induced by exercise depends on exercise intensity in elderly patients with COPD. However, it is very difficult to clinically determine this point, especially in elderly patients.

\section{Conclusions}

In summary, the results of this study showed that DAS reduced the perception of dyspnea induced by low-intensity, constant-load exercise in elderly subjects with COPD. Our results suggest that DAS is a non-pharmacologic therapy that can be used to reduce dyspneic sensation in elderly patients with COPD.

\section{ACKNOWLEDGMENTS}

We thank Dr Sumihisa Honda (Nagasaki University Graduate School of Biomedical Sciences, Nagasaki, Japan) for statistical advice and $\mathrm{Mr}$ Thomas Mandeville (New Day School, Sendai, Japan) for reading the manuscript.

\section{REFERENCES}

1. Shingai K, Kanezaki M. Effect of dyspnea induced by breath-holding on maximal muscular strength of patients with COPD. J Phys Ther Sci 2014;26(2):255-258.

2. von Leupoldt A, Taube K, Schubert-Heukeshoven S, Magnussen H, Dahme B. Distractive auditory stimuli reduce the unpleasantness of dyspnea during exercise in patients with COPD. Chest 2007;132(5): 1506-1512.

3. Bauldoff GS, Hoffman LA, Zullo TG, Sciurba FC. Exercise maintenance following pulmonary rehabilitation: effect of distractive stimuli. Chest 2002;122(3):948-954.

4. American Thoracic Society, American College of Chest Physicians. ATS/ACCP statement on cardiopulmonary exercise testing. Am J Respir Crit Care Med 2003;167(2):211-277.

5. Luxton N, Alison JA, Wu J, Mackey MG. Relationship between field walking tests and incremental cycle ergometry in COPD. Respirology 2008;13(6):856-862.

6. Murray JA, Waterman LA, Ward J, Baird JC, Mahler DA. Perceptual and physiologic responses during treadmill and cycle exercise in patients with COPD. Chest 2009;135(2):384-390.

7. Castro AA, Porto EF, Iamonti VC, de Souza GF, Nascimento OA, Jardim JR. Oxygen and ventilatory output during several activities of daily living performed by COPD patients stratified according to disease severity. PLoS ONE 2013;8(11):e79727. 


\section{EfFect of DAS on Dyspnea in Elderly Subjects With COPD}

8. Yamashita S, Iwai K, Akimoto T, Sugawara J, Kono I. Effect of music during exercise on RPE, heart rate and the autonomic nervous system. J Sports Med Phys Fitness 2006;46(3):425-430.

9. Davenport PW, Vovk A. Cortical and subcortical central neural pathways in respiratory sensations. Respir Physiol Neurobiol 2009;167(1): 72-86.

10. von Leupoldt A, Dahme B. Cortical substrates for the perception of dyspnea. Chest 2005;128(1):345-354.

11. von Leupoldt A, Sommer T, Kegat S, Baumann HJ, Klose H, Dahme $\mathrm{B}$, Büchel C. The unpleasantness of perceived dyspnea is processed in the anterior insula and amygdala. Am J Respir Crit Care Med 2008;177(9):1026-1032.

12. Eldridge FL, Millhorn DE, Waldrop TG. Input-output relationships of the central respiratory controller during peripheral muscle stimulation in cats. J Physiol 1982;324:285-295.

13. Barbaro MP, Lacedonia D, Palladino GP, Bergantino L, Ruggeri C, Martinelli D, Carpagnano GE. Dyspnea perception in asthma: role of airways inflammation, age and emotional status. Respir Med 2011; 105(2):195-203

14. Tun PA, Williams VA, Small BJ, Hafter ER. The effects of aging on auditory processing and cognition. Am J Audiol 2012;21(2):344-350.

15. Rabe KF, Hurd S, Anzueto A, Barnes PJ, Buist SA, Calverley P, et al. Global strategy for the diagnosis, management, and prevention of chronic obstructive pulmonary disease: GOLD executive summary. Am J Respir Crit Care Med 2007;176(6):532-555.

16. Kanezaki M, Ebihara, Nikkuni E, Gui P, Suda C, Ebihara T, et al. Perception of urge-to-cough and dyspnea in healthy smokers with decreased cough reflex sensitivity. Cough 2010;6(1):1.

17. Thornby MA, Haas F, Axen K. Effect of distractive auditory stimuli on exercise tolerance in patients with COPD. Chest 1995;107(5): 1213-1217.

18. Pfister T, Berrol C, Caplan. Effects of music on exercise and perceived symptoms in patients with chronic obstructive pulmonary disease. J Cardiopulm Rehabil 1998;18(3):228-232. 